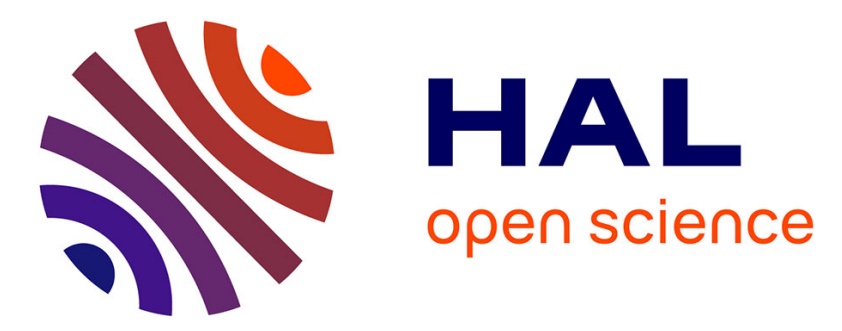

\title{
Corrigendum to "Design, analysis and validation of a simple dynamic model of a submerged membrane bioreactor"
}

\author{
Guilherme Araujo Pimentel, Alain Vande Wouver, Jérôme Harmand, Alain \\ Rapaport
}

\section{To cite this version:}

Guilherme Araujo Pimentel, Alain Vande Wouver, Jérôme Harmand, Alain Rapaport. Corrigendum to "Design, analysis and validation of a simple dynamic model of a submerged membrane bioreactor". Water Research, 2016, 89, pp.384-385. 10.1016/j.watres.2015.10.044 . hal-02638986

\section{HAL Id: hal-02638986 https://hal.inrae.fr/hal-02638986}

Submitted on 28 May 2020

HAL is a multi-disciplinary open access archive for the deposit and dissemination of scientific research documents, whether they are published or not. The documents may come from teaching and research institutions in France or abroad, or from public or private research centers.
L'archive ouverte pluridisciplinaire HAL, est destinée au dépôt et à la diffusion de documents scientifiques de niveau recherche, publiés ou non, émanant des établissements d'enseignement et de recherche français ou étrangers, des laboratoires publics ou privés. 


\section{Accepted Manuscript}

Corrigendum to "Design, analysis and validation of a simple dynamic model of a submerged membrane bioreactor"

Guilherme A. Pimentel, Alain Vande Wouwer, Jérôme Harmand, Alain Rapaport

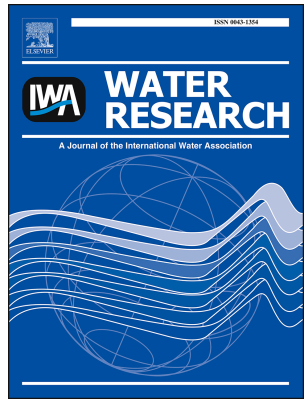

PII:

S0043-1354(15)00380-2

DOI:

10.1016/j.watres.2015.10.044

Reference: WR 11605

To appear in: Water Research

Please cite this article as: Pimentel, G.A., Wouwer, A.V., Harmand, J., Rapaport, A., Corrigendum to "Design, analysis and validation of a simple dynamic model of a submerged membrane bioreactor", Water Research (2015), doi: 10.1016/j.watres.2015.10.044.

This is a PDF file of an unedited manuscript that has been accepted for publication. As a service to our customers we are providing this early version of the manuscript. The manuscript will undergo copyediting, typesetting, and review of the resulting proof before it is published in its final form. Please note that during the production process errors may be discovered which could affect the content, and all legal disclaimers that apply to the journal pertain. 


\title{
Corrigendum to "Design, analysis and validation of a simple dynamic model of a submerged membrane bioreactor"
}

\author{
Guilherme A. Pimentela,b,d, Alain Vande Wouwera, Jérôme Harmand ${ }^{\mathrm{b}, \mathrm{c}}$, Alain \\ Rapaport ${ }^{\mathrm{b}, \mathrm{d}}$ \\ aUniversity of Mons - Automatic Control Laboratory - Biosys, Boulevard Dolez 31, 7000 Mons, Belgium \\ ${ }^{b}$ Equipe Projet INRA-INRIA MODEMIC, Route des Lucioles 06902 Sophia-Antipolis, France \\ 'INRA, UR050, Laboratoire de Biotechnologie de l'Environnement, Av. des Etangs, F-11100 \\ Narbonne, France \\ dUMR INRA-SupAgro MISTEA, 2 Place Viala 34060 Montpellier, France
}

\begin{abstract}
In this paper, the readers can find the corrections of the published article. The authors apologize for any inconvenience caused.
\end{abstract}

\section{SECTION 4.2 - Three-time-scale singular perturbation}

The small parameters are assumed to be $\epsilon_{1}=|\gamma|$ and $\epsilon_{2}=\frac{1}{V}$.

Hypothesis 1. $\gamma$ is small.

Hypothesis 2. The volume $V$ is large, with $V<\frac{1}{|\gamma|}$.

The application of the procedure introduced in the previous subsection yields:

Email addresses: guilherme.araujopimentel@umons.ac.be (Guilherme A. Pimentel), alain.vandewouwer@umons.ac.be (Alain Vande Wouwer),

jerome.harmandesupagro.inra.fr

(Jérôme Harmand), rapaport@supagro.inra.fr (Alain Rapaport) 
First: The stretched time-scale $\tau_{1}=\epsilon_{1} t \rightarrow \frac{1}{d t}=\frac{\epsilon_{1}}{d \tau_{1}}$

$$
\begin{aligned}
& \frac{d x_{s l}}{d \tau_{1}}=\operatorname{sign}(\gamma) x_{s l} \\
& \epsilon_{1} \frac{d y_{1}}{d \tau_{1}}=g_{1}\left(y_{1}, y_{2}\right)=-\frac{1}{Y} \mu\left(y_{1}\right) y_{2}+\frac{Q_{\text {in }}}{V}\left(S_{\text {in }}-y_{1}\right) \\
& \epsilon_{1} \frac{d y_{2}}{d \tau_{1}}=g_{2}\left(x_{s l}, y_{1}, y_{2}, z\right)= \\
& \mu\left(y_{1}\right) y_{2}+\frac{Q_{\text {in }}}{V} X_{\text {in }}-\frac{Q_{\text {in }}}{V} y_{2}+x_{s l} \frac{J_{\text {air }}}{V} \frac{z^{2}}{K_{\text {air }}+z} \quad \text { (Fast) } \\
& \epsilon_{1} \frac{d_{z}}{d \tau_{1}}=h\left(x_{s l}, y_{2}, z\right)=Q_{\text {cake }} y_{2}-x_{s} J_{\text {air }} \frac{z^{2}}{K_{\text {air }}+z}
\end{aligned}
$$

Considering $Q_{w}=Q_{i n}-Q_{c a k e}$ and letting $\bar{S}_{i n}=S_{i n}+\frac{X_{i n}}{Y}>S_{i n}$ the following slow-fast approximation can be developed:

$$
\begin{gathered}
0=g_{1}\left(y_{1}, y_{2}\right) \rightarrow \mu\left(y_{1}\right)=\frac{Q_{w}}{V} \frac{S_{i n}-y_{1}}{\bar{S}_{i n}-y_{1}} \\
0=g_{2}\left(x_{s l}, y_{1}, y_{2}, z\right) \rightarrow y_{2} \frac{Q_{i n}\left(\bar{S}_{i n}-Y y_{1}\right)}{Q_{w}} \\
0=h\left(x_{s l}, y_{2}, z\right) \rightarrow \frac{x_{\text {sl }} J_{\text {air }} z^{2}}{V\left(K_{\text {air }}+z\right)}=Q_{\text {out }} y_{2}
\end{gathered}
$$

Second: The stretched time-scale $\tau_{2}=\epsilon_{2} \tau_{1}=\epsilon_{2} \epsilon_{1} t \rightarrow \frac{1}{d \tau}=\frac{\epsilon_{2}}{d \tau_{2}}$ and $x_{s l}$ is constant.

$$
\begin{aligned}
& \epsilon_{1} \frac{d y_{1}}{d \tau_{2}}=-\frac{1}{Y \epsilon_{2}} \mu\left(y_{1}\right) y_{2}+Q_{i n}\left(S_{i n}-y_{1}\right) \\
& \epsilon_{1} \frac{d y_{2}}{d \tau_{2}}=\left(\frac{\mu\left(y_{1}\right)}{\epsilon_{2}}-Q_{w}\right) y_{2}+Q_{i n} X_{\text {in }}-Q_{\text {cake }} y_{2}+x_{s l} J_{\text {air }} \frac{z^{2}}{K_{\text {air }}+z} \\
& \epsilon_{2} \epsilon_{1} \frac{d z}{d \tau_{2}}=h\left(x_{s l}, y_{2}, z\right)=Q_{\text {cake }} y_{2}-x_{s l} J_{\text {air }} \frac{z^{2}}{K_{\text {air }}+z} \\
& 0=h\left(x_{s l}, y_{2}, z\right) \rightarrow z=\frac{\frac{Q_{c a k e} Q_{i n} Y \bar{s}_{i n}}{Q_{w}}+\sqrt{Q_{\text {cake }}^{2}\left(\frac{Q_{i n} Y \bar{S}_{i n}}{Q_{w}}\right)^{2}+4 \frac{Q_{c a k e} Y \bar{s}_{i n} K_{\text {air }} J_{a i r} x_{s l} Q_{i n}}{Q_{w}}}}{2 x_{s l} J_{\text {air }}}
\end{aligned}
$$

\section{TABLE 2}


Table 2: Parameters with their confidence interval.

\begin{tabular}{|c|c|c|c|c|}
\hline & Ultrafast [0.02 Days] & Fast [6 Days] & Slow [20 Days] & All Parameters \\
\hline$\beta_{0}$ & $(4.819 \pm 0.573) \times 10^{4}$ & fixed value & fixed value & $(5.531 \pm 0.643) \times 10^{4}$ \\
\hline$K_{\text {air }}$ & $(4.773 \pm 0.575) \times 10^{1}$ & fixed value & fixed value & $(4.596 \pm 0.134) \times 10^{1}$ \\
\hline$Y$ & fixed value & $(8.996 \pm 0.022) \times 10^{-1}$ & fixed value & $(8.985 \pm 0.016) \times 10^{-1}$ \\
\hline$\mu_{S, \max }$ & fixed value & $(2.004 \pm 0.722)$ & fixed value & $(2.265 \pm 0.343)$ \\
\hline$\gamma$ & fixed value & fixed value & $(0.001 \pm 0.882)$ & $(0.001 \pm 0.535)$ \\
\hline $\min \left(f_{\text {cost }}(\theta)\right)$ & 53.55 & 0.8592 & 6.42 & 22.79 \\
\hline
\end{tabular}

